\title{
Estratégias que viabilizam o acesso aos serviços de Atenção Primária à Saúde no Reino Unido
}

\author{
Strategies that enable access to Primary Health Care services in the United Kingdom \\ Estrategias que viabilizan el acceso a los servicios de Atención Primaria de Salud en \\ Reino Unido
}

\author{
Armando Henrique Norman ${ }^{1} \odot$ \\ ${ }^{1}$ Universidade Federal de Santa Catarina (UFSC). Florianópolis, SC, Brasil.
}

\section{Resumo}

\begin{abstract}
Este artigo apresenta algumas características organizativas e operacionais da Atenção Primária à Saúde (APS) inglesa. Seu objetivo é evidenciar pontos relevantes no funcionamento das clínicas da APS que facilitam o equilíbrio dinâmico entre o atendimento à demanda espontânea e as ações programáticas em saúde. Ele tem como base um estudo etnográfico, realizado no Reino Unido no período de $2013 / 14$, no qual se analisou o modelo de pagamento por desempenho no país. Os tópicos abordados incluem: (a) composição das equipes da APS inglesa; (b) organograma funcional de uma clínica de APS; (c) consulta de 10 minutos dos médicos de família e horário de funcionamento da clínica; (d) recepção; (e) equipe de enfermagem; e (f) sistema de tecnologia da informação. Cada um desses itens possui referências para as quais é possível explorar websites e tutoriais de modo a compreender melhor alguns aspectos das clínicas da APS inglesa. O Reino Unido profissionalizou o acesso dos pacientes aos serviços da APS. Isto ocorreu por meio de um contingente maior de pessoal administrativo, menor proporção população/médico de família, maior autonomia da equipe de enfermagem e um robusto sistema de tecnologia da informação. No Brasil, a Estratégia Saúde da Família (ESF) necessita percorrer caminho semelhante a fim de aprimorar a qualidade da APS no país.
\end{abstract}

Palavras-chave: Medicina de Família e Comunidade; Atenção Primária à Saúde; Estratégia Saúde da Família; Acesso Universal aos Serviços de Saúde; Acesso dos Pacientes aos Registros

Como citar: Norman AH. Estratégias que viabilizam o acesso aos serviços de Atenção Primária à Saúde no Reino Unido. Rev Bras Med Fam Comunidade. 2019;14(41):1945. https://doi.org/10.5712/rbmfc14(41)1945
Autor correspondente: Armando Henrique Norman. E-mail: ahnorman@hotmail.com Fonte de financiamento: declara não haver.

Parecer CEP: não se aplica.

Procedência e revisão por pares: revisado por pares. Recebido em: 25/03/2019. Aprovado em: 27/04/2019. 


\begin{abstract}
This article presents some organizational and operational characteristics of UK Primary Health Care (PHC). The objective is to show relevant points in the functioning of $\mathrm{PHC}$ clinics that facilitate the dynamic balance between same-day appointments and programmatic health actions. It derives from empirical material of an ethnographic study, conducted in the United Kingdom in the period of 2013/14, which analyzed the pay-for-performance mode in the country. The issues covered include: (a) composition of English PHC teams; (b) PHC clinic functional organogram; (c) 10 minute consultation of family physician and clinic opening hours; (d) reception; (e) nursing team; and (f) information technology system. The supporting references allow to simultaneously explore websites and tutorials to better understand some aspects of the English PHC dynamics. The UK has professionalized patients' access to PHC. This has entailed larger number of administrative personnel, a smaller population/family physician ratio, greater autonomy of the nursing team and a robust information technology system. In Brazil, the Family Health Strategy (FHS) needs to follow a similar path in order to improve the primary healthcare quality in the country.
\end{abstract}

Keywords: Family Practice; Primary Health Care; Family Health Strategy; Universal Access to Health Care Services; Patient Access to Records

\title{
Resumen
}

Este artículo presenta algunas características organizativas y operativas de la atención primaria de salud (APS) en el Reino Unido. El objetivo es presentar los puntos relevantes en las clínicas de la APS que articulados facilitan el equilibrio dinámico entre citas el mismo día y acciones programáticas de salud. Tiene como base material empírico de un estudio etnográfico, realizado en el Reino Unido en el período de 2013/14, que analizó el modelo de pago por desempeño en el país. Los temas cubiertos incluyen: (a) la composición de los equipos de APS inglesa; b) organograma funcional de una clínica de APS; (c) consulta de 10 minutos consulta del médico de familia y horario de funcionamiento de las clínicas de APS; (d) recepción; (e) equipo de enfermería; y (f) sistema de tecnología de la información. Las referencias de apoyo permiten explorar simultáneamente webpages y tutoriales para comprender mejor algunos aspectos de la dinámica de la APS. El Reino Unido ha profesionalizado el acceso de los pacientes a la APS. Esto ha implicado un mayor número de personal administrativo, una proporción menor de población/médicos de familia, una mayor autonomía del equipo de enfermería y un sistema robusto de tecnología de la información. En Brasil, la Estrategia de Salud Familiar (ESF) necesita seguir un camino similar para mejorar la calidad de la APS en el país.

Palabras clave: Medicina Familiar y Comunitaria; Atención Primaria de Salud; Estrategia de Salud Familiar; Acceso Universal a los Servicios de Salud; Acceso de los Pacientes a los Registros

\section{Introdução}

A expansão da Estratégia Saúde da Família (ESF) ampliou enormemente o acesso aos serviços de atenção primária no Sistema Único de Saúde (SUS) brasileiro. Entretanto, o acesso nas clínicas de Atenção Primária à Saúde (APS) ainda carece de regulamentação e aprimoramento. ${ }^{1}$ Barreiras ao acesso prejudicam a coordenação longitudinal e personalizada dos cuidados de uma coorte de indivíduos em um determinado território. Essas barreiras podem ser de várias ordens: geográficas, financeiras, estruturais e organizativas dos processos de trabalho. Portanto, eliminar ou minimizar os obstáculos do acesso aos serviços da APS é base para a operacionalização da totalidade dos seus atributos. ${ }^{2}$

O presente artigo faz uma reflexão sobre algumas características organizativas do processo de trabalho das clínicas da APS inglesa, que favorecem o equilíbrio dinâmico do acesso, tanto à demanda espontânea quanto às ações programáticas em saúde. Ele tem como base um estudo etnográfico, publicado em 2016, no qual foi analisado o impacto do pagamento por desempenho sobre a APS do Reino Unido. ${ }^{3}$

Primeiramente, apresenta-se um breve histórico de como se organizou o National Health Service (NHS) para garantir o acesso da população aos cuidados médicos. Em seguida, são descritas algumas características operacionais e funcionais das clínicas da APS inglesa. Dessa forma, espera-se contribuir para o debate sobre a melhoria do acesso nos serviços da APS brasileira. 


\section{Um breve histórico do NHS}

Em 5 de julho 2018, o NHS celebrou 70 anos de existência. O NHS continua sendo considerado um dos melhores sistemas de saúde universais do mundo. ${ }^{4} \mathrm{Um}$ dos seus eixos organizativos ocorre por meio de uma APS abrangente. As clínicas de APS (General Practices) garantem tanto o acesso quanto a coordenação do cuidado de uma coorte de indivíduos em um determinado território. Um questionamento que surge: como foi possível universalizar o acesso aos serviços de saúde para 50 milhões de britânicos em um intervalo de seis meses da criação do NHS, em 1948?

O NHS não surge em um vácuo. O documentário "The NHS: a difficult beginning" evidencia esse fato. ${ }^{5}$ Em 1911, o National Insurance Act garantia aos trabalhadores britânicos o direito aos serviços de saúde. ${ }^{6}$ Estavam excluídos desse tipo de seguridade social crianças, donas de casa, desempregados, aposentados e idosos. Em 1920, o relatório Dawson estabelecia as bases para uma reorganização do sistema de saúde no país e cunhava o termo atenção primária à saúde. ${ }^{7}$

A Segunda Guerra Mundial teve uma influência importante na participação do Estado no provimento de serviços médicos como estratégia para o enfrentamento dos problemas causados pelos bombardeios alemães (Blitz). Assim, no pós-guerra havia uma sede por mudança e o plano de governo do partido trabalhista (Labour Party) era o que mais atendia às demandas da população na construção de um estado de bem-estar social (Welfare State). Esse ideal estava materializado no Report on Social Insurance and Allied Services, em que William Beveridge documenta o modo como o estado deveria funcionar para melhor atender às necessidades de sua população. Nascia, assim, a moderna seguridade social. ${ }^{8}$

Com a vitória dos trabalhistas no pós-guerra, Aneurin Bevan, o então ministro da saúde, propõe ao parlamento a criação do NHS em 5 de julho de $1948 .{ }^{8}$ Um dos principais obstáculos à criação do NHS foram os médicos representados pela British Medical Association (BMA). A BMA lançou uma votação nacional para saber se os médicos eram a favor ou contra o NHS. Apenas $13 \%$ foram favoráveis à nacionalização dos serviços de saúde. Entretanto, a pesquisa de opinião pública evidenciava que mais de $70 \%$ da população apoiava a sua criação.

O governo investia no esclarecimento da população sobre a importância do NHS. ${ }^{9}$ Bevan conseguiu reverter a posição dos médicos, ao garantir aos especialistas focais que poderiam manter suas atividades privadas, caso assim o desejassem, além de poderem trabalhar como assalariados dos hospitais públicos. Os General Practitioners (GPs) foram a última resistência a ser vencida. Os GPs temiam perder sua autonomia ao se tornarem servidores públicos.

Havia também o risco financeiro, visto que a maioria operava como um pequeno negócio e temia que os investimentos feitos na clínica fossem perdidos. Esse contexto fez com que Bevan, em vez de criar uma carreira de estado para os GPs, contratasse diretamente os serviços dos GPs, que passaram a ter o status de contratantes independentes (independent contractors).

Com essa manobra, a BMA lançou uma nova pesquisa que apontava que $40 \%$ dos GPs passaram a apoiar a criação do NHS. Assim, na batalha pela criação do NHS, o governo, por meio da campanha midiática "Choose your doctor now!" solicitava que as pessoas perguntassem ao seu GP se ele iria participar do NHS. ${ }^{10}$ Caso positivo, todos os membros da família teriam que se registrar com o seu GP. 
Essa estratégia fez com que muitos dos GPs aderissem à proposta de criação de um serviço universal de saúde, com receio de ficar sem clientes. Portanto, quando nasce o NHS, a universalização do acesso aos serviços da APS ultrapassava os $80 \%$ de cobertura. O pragmatismo de Bevan fortaleceu simultaneamente o acesso e o vínculo por meio da responsabilização nominal de um GP pela prestação 24/7 (24 horas/sete dias por semana) dos serviços de APS.

Esse status de contratante independente permanece até os dias de hoje como pedra angular do modelo de APS britânico. ${ }^{6}$ Certamente, a opção pelo contrato independente trouxe alguns problemas, tanto para os GPs quanto para o governo. Por exemplo, havia uma inseguridade financeira para os GPs, causada pelo modelo de capitação. Do lado do governo, esse modelo produziu uma grande variação no padrão assistencial prestado pelos GPs.

O mecanismo encontrado para fazer os ajustes necessários a essa modalidade de prestação de serviços foi o detalhamento dos contratos pactuados entre BMA e governo. Por exemplo, o contrato de 1966 conseguiu estabelecer o máximo de 2000 pessoas por GP, além de injetar dinheiro para a contratação de pessoal e favorecer a formação de grupos médicos, isto é, mais de dois GPs trabalhando juntos em associação.

O NHS sofreu grandes transformações ao longo da sua história, principalmente durante o governo de Margaret Thatcher. Thatcher introduziu a lógica de "mercado interno" dentro do setor público, fragmentando o setor da saúde entre provedores e compradores de serviços. ${ }^{11}$ Por exemplo, os "GP- fundholders" e as autoridades distritais (District Health Authorities) passaram a ser compradores de serviços. Os primeiros eram responsáveis por comprar principalmente os serviços de nível secundário (especialidades focais); os segundos eram responsáveis por avaliar as necessidades em saúde da população e comprar serviços hospitalares e comunitários. Eles passaram a receber recursos financeiros diretamente do Departamento de Saúde.

Desse modo, os provedores foram obrigados a competir por esses recursos financeiros por meio da prestação de serviços com base em contratos comerciais. ${ }^{12} \mathrm{Ou}$ seja, os hospitais públicos foram transformados em fundações (trusts) e passaram a receber pelos serviços prestados (ou "comprados") pelos provedores, em vez de simplesmente demandarem do governo recursos financeiros. Acreditava-se que uma competição interna entre os diversos provedores seria saudável para reduzir os custos e melhorar a qualidade dos serviços dentro do sistema.

O "gerencialismo" também foi uma inovação do governo Thatcher com seus ideais de metas, resultados e de custo-efetividade. A ideologia de mercado passou a influenciar intensamente a dinâmica da APS britânica, mas ao contrário do que se esperava, os custos do setor aumentaram. ${ }^{11}$ Essa fragmentação permaneceu como parte integrante do NHS, sendo inclusive aprimorada durante a gestão do primeiro-ministro trabalhista Tony Blair com a criação dos Primary Care Trusts (PCTs). O PCT basicamente integrou as funções do GP- fundholders e do District Health Authorities, mantendo a fragmentação entre comprador/provedor. ${ }^{12}$

É nesse contexto que surge o contrato de 2004 no qual foram estabelecidas três faixas de pagamento de repasse aos GPs: (1) Capitação 60\%; (2) Pagamento por desempenho 25\% (Quality and Outcomes Framework - QOF); e (3) Serviços adicionais (15\%). Os contratos passaram a estabelecer a carteira de serviços que cada clínica de APS ofereceria. Desde 2004, essas proporções têm sofrido reajustes, havendo uma redução importante nos valores provenientes do pagamento por desempenho. ${ }^{3}$ 
Particularmente, o contrato de 2004 simboliza uma tendência nas economias neoliberais em que a "saúde" é vista como mercadoria. Essa mercantilização da saúde vem favorecendo a fragmentação e a despersonalização do cuidado na APS britânica. ${ }^{3,13}$ Esse processo segue se aprofundando desde o retorno do Partido Conservador ao poder. A partir de 2010, o governo do primeiro-ministro David Cameron fomenta uma maior participação do setor privado no provimento de serviços ao transformar os PCTs em Clinical Commissioning Groups (CCGs), reforçando a lógica de mercado dentro do NHS. ${ }^{12}$

Apesar das forças mercadológicas e dos GPs se manterem como contratantes independentes, a seguridade social dos GPs (aposentadoria) segue sendo vinculada ao NHS que, em tese, seria apenas um privilégio dos servidores públicos. Essa mescla de contratante independente e de assegurado do Estado faz toda a diferença no plano de carreira desses profissionais. O pragmatismo de Bevan em garantir acesso a um GP foi um lance de mestre. A partir desse momento, foi possível buscar soluções para as partes envolvidas por meio dos contratos.

O modelo de contrato independente do Reino Unido não significa uma terceirização, porque os recursos continuam públicos. Exige-se transparência, auditorias e prestação de contas, como explicitado na webpage de uma das clínicas: "The NHS England requires that the net earnings of doctors engaged in the practice is publicised and the required disclosure is shown below", ${ }^{14}$ ou seja, ao final das webpages de cada clínica da APS está disponível o ganho médio dos GPs. Não apenas se presta conta dos contratos e gastos em serviços, mas dos salários de quem participa do contrato.

Isso não ocorre na relação público-privado no Brasil, via modelo das organizações sociais (OS), por exemplo, que estão sendo implantadas na APS brasileira, em que a "produção do excedente é apropriada na forma de altos salários para os dirigentes e/ou proprietários de empresas" (p. 5). ${ }^{15}$

\section{Composição das clínicas da APS Inglesa}

A organização inicial do NHS foi denominada como tripartite: (a) serviços hospitalares regionalizados; (b) serviços comunitários; e (c) serviços médicos, odontológicos e farmacêuticos na APS. Os serviços comunitários incluíam cuidado pré-natal, cuidados de enfermagem, puericultura, imunização e promoção da saúde, que ficavam a cargo da autoridade local de saúde. Os GPs mantiveram seu contrato independente, vinculados ao conselho executivo, por meio do repasse central de recursos. De certa forma, perdura até hoje a divisão do provimento entre serviços comunitários e serviços de cuidados assistenciais de médicos e enfermeiros. ${ }^{8,16}$

Em outras palavras, nas clínicas da APS inglesa, o provimento de serviços ocorre por meio de funcionários contratados diretamente pela clínicas da APS e pelo apoio de funcionários contratados pelo distrito sanitário e/ou via Clinical Commissioning Group (CCG). ${ }^{17}$ Por exemplo, os médicos de família, a equipe de enfermagem e o pessoal administrativo são contratados pela clínica da APS.

Entretanto, está sob a responsabilidade da autoridade local (via distrito sanitário ou CCG) todo um conjunto de profissionais, tais como: (a) district nurses - enfermeiras distritais: responsáveis por grande parte do cuidado domiciliar, i.e. curativos, verificação de pressão arterial, coleta de exames, avaliação funcional e de saúde, etc; (b) midwives - enfermeiras obstetras encarregadas de todo o pré-natal de risco habitual; ${ }^{18}$ (c) health visitors - toda puericultura é realizada pelas enfermeiras que cuidam das crianças de 
0-5 anos; (d) school nurses - enfermeiras que cuidam das crianças em idade escolar (acima de 5 anos), quer nas escolas ou nas clínicas da APS; (e) Counsellors - cuidados em saúde mental são providos por profissionais de nível médio, com consultas de 30 a 60 minutos; (f) nutricionistas; (g) podólogos - esses dois últimos estão principalmente envolvidos no cuidados aos pacientes diabéticos.

O fato de gestantes e crianças de risco habitual serem cuidadas em um espaço próprio favorece um olhar menos medicalizante. Contudo, quando esses espaços não estão disponíveis, as instalações das clínicas da APS servem de estrutura para o provimento desses cuidados. Existem outros apoios às clínicas da APS, via terceiro setor, tais como: equipes de cuidados paliativos (Macmillan Nurses),${ }^{19}$ equipes de saúde mental, ${ }^{20}$ cursos e apoio ao cuidado do diabetes, ${ }^{21}$ e assim por diante.

As clínicas da APS britânicas trabalham em base territorial, possuindo uma área de abrangência pela qual a clínica é responsável. O contrato de 2004 tornou facultativo aos GPs terem ou não uma lista pessoal de pacientes. Os pacientes passaram a ter seu vínculo com a clínica e não com um GP em particular. No mesmo contrato, os GPs puderam se desobrigar dos cuidados 24/7 de seus pacientes. Ficou a cargo dos PCTs (atualmente CCGs) a responsabilidade de organizar o acesso após o horário de funcionamento das clínicas, os chamados out of hours services. ${ }^{3}$

\section{Organograma das clínicas da APS Inglesa}

Para exemplificar a estrutura funcional da APS inglesa, utilizarei os dados da etnografia, de modo a ilustrar o que já foi exposto. ${ }^{3}$ Em uma das clínicas da APS em que realizei a pesquisa de campo, a cobertura populacional era de aproximadamente 15.500 pessoas em uma área semirrural espalhada em quatro comunidades. Assim, havia uma clínica matriz no povoado principal e três outras clínicas filiais menores. Estas eram distantes aproximadamente cinco minutos, de carro, umas das outras.

AFigura 1 ilustra o organograma desse grupo médico. Notem que os sócios (todos médicos de família) estão no topo da hierarquia. Logo abaixo, estão o gerente geral (practice manager) e o gerente financeiro/ comercial. Esses gerentes são apoiados por um auxiliar administrativo, além de supervisionarem seis funcionários administrativos. Essa equipe de administrativos é responsável por todo o fluxo de informação da clínica (referência e contrarreferência, marcações de exames e seus resultados, digitalização de exames para serem anexados ao prontuário dos pacientes, assim como pelo registro dos pacientes, isto é, vinculação e desvinculação dos pacientes à clínica).

Abaixo do gerente geral, estão a supervisora das recepcionistas (um total de oito recepcionistas + três supervisoras para cada uma das filiais) e a enfermeira-chefe (lead nurse), responsável pela coordenação de oito enfermeiras e três técnicos de enfermagem (health care assistants). Os números entre parênteses ao lado de cada categoria profissional representam a quantidade desses profissionais na clínica estudada. A linha tracejada à direita do gerente geral indica os profissionais "anexados/contratados". Esse é o braço comunitário provido pelo distrito sanitário que utiliza as instalações das clínicas para atender a comunidade.

Em relação aos profissionais médicos, existem três GPs assalariados. Estes são contratados pela clínica, visto que alguns dos GPs associados desempenham outras atividades fora da clínica e sua ausência necessita ser compensada. Por exemplo, se um dos GPs trabalha algumas horas na gestão no CCG, a clínica é indenizada para prover uma equivalência de horas médico-assistenciais. 


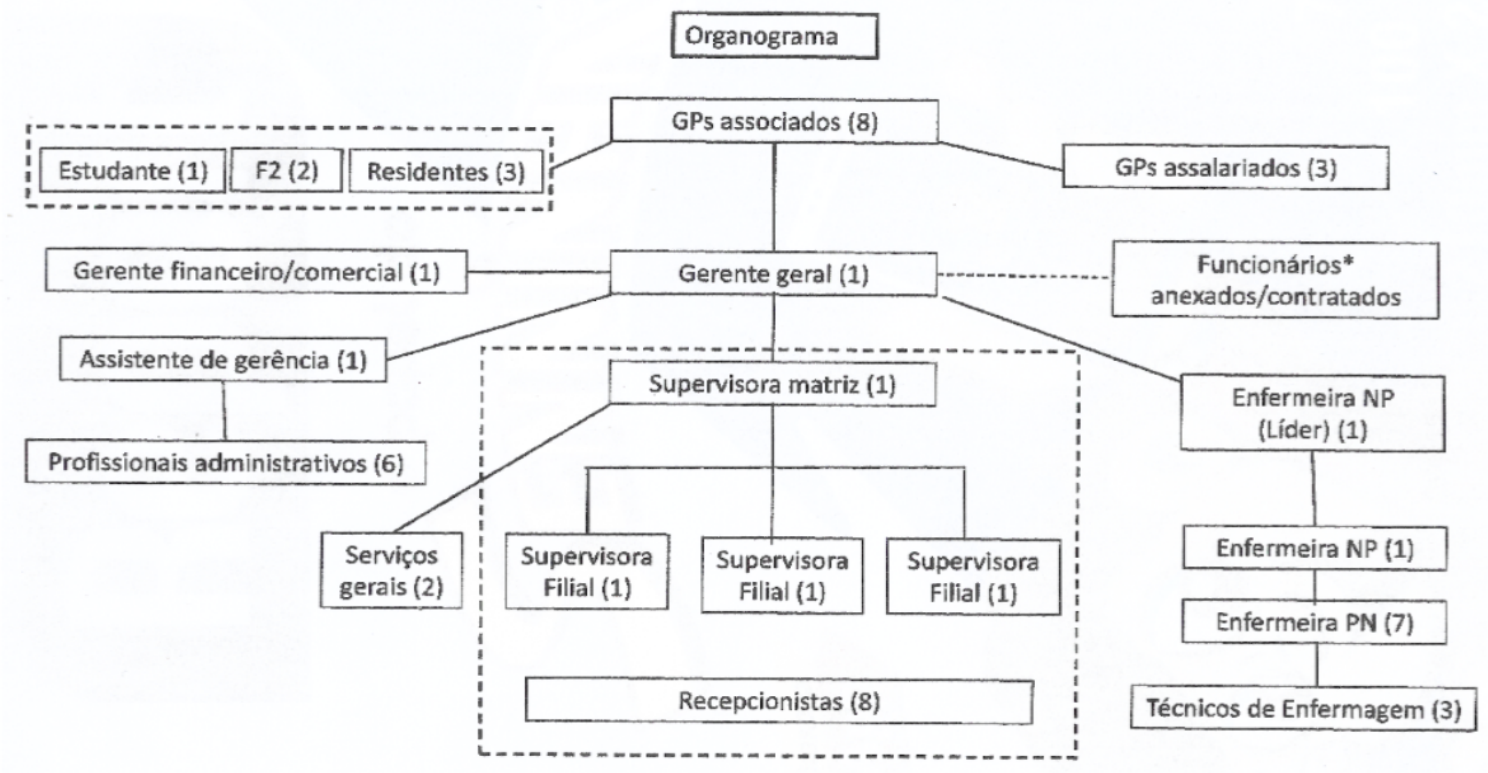

Figura 1. Organograma de uma clínica da APS inglesa para uma população de aproximadamente 15.500 pessoas. Os números entre parênteses ao lado das categorias profissionais representam o seu quantitativo ( ${ }^{\star}$ Enfermeiras parteiras, distritais, de puericultura, podologistas e nutricionistas).

Outro exemplo corresponde às liberações dos GPs para atividade acadêmica dos residentes, em que o NHS reembolsa financeiramente a clínica para não prejudicar a assistência médica da comunidade. Existem também médicos temporários (denominados de locums) que cobrem eventuais ausências, mas esses estão incluídos no orçamento das clínicas da APS. É o caso de cobertura das férias de profissionais ou licença-saúde.

O acesso facilitado na APS britânica está, certamente, dependente da proporção adequada entre número de GPs e população de abrangência. Em média, cada GP tem uma lista de pacientes de 1800 pessoas. Entretanto, outros fatores relativos ao processo de trabalho também favorecem o acesso.

\section{Políticas institucionais}

Como parte do contrato de 2004, as clínicas de APS inglesas tiveram que ofertar em 48 horas uma consulta médica e em 24 horas uma avaliação pela equipe de enfermagem..$^{22}$ Isso aumentou o acesso, mas quebrou a fidelização. Em outras palavras, o acesso a qualquer médico foi facilitado, mas não o acesso a um profissional médico específico. Entretanto, no contrato de 2015 o governo inglês buscou reverter essa tendência exigindo que todos os pacientes vinculados às clínicas da APS tivessem um GP nomeado.

$\mathrm{Na}$ verdade, existe uma tendência de flexibilizar o cuidado personalizado em situações agudas, deixando mais a cargo dos usuários a escolha e a valorização desse processo de cuidados continuados. ${ }^{23}$ Esse entendimento pode ser observado em diferentes grupos médicos no modo como informam a seus 
usuários, por exemplo: Linthorpe Surgery informa que o "Named GP will be responsible for your overall care at the practice, however this does not prevent you from seeing any other doctor at the surgery. If you have not already been informed of your named GP and would like to know who this is, please contact the surgery." Enquanto o Marsh House Medical Practice tem uma postura mais clara, ao afirmar que "We offer an individual list system at Marsh House Medical Practice whereby the patients are seen by their own GP. The partners feel this offers patient's continuity of care. You may however, request to see an alternative doctor in special circumstances and where possible the practice will try to accommodate."24

Assim, atualmente na Inglaterra coexistem clínicas que permanecem com listas pessoais e aquelas que flexibilizaram esse atendimento continuado personalizado. Os prontuários eletrônicos têm sido um dos principais aliados nesse processo, visto que melhoram a qualidade e a segurança dos registros clínicos e permitem que a continuidade do cuidado possa ser oferecida por outro membro da equipe de saúde. Portanto, desde 2004 os cuidados continuados e personalizados foram afetados, em certa medida, pela desobrigação dos cuidados 24/7, pelas exigências de acesso em 48 horas a um médico, pela vinculação à clínica (e não a um GP em particular) e pelos processos de informatização.

As clínicas da APS funcionam em um horário muito semelhante ao brasileiro, com algumas variações. Não há uma regra e cada clínica pode flexibilizar os horários de funcionamento de acordo com o perfil comunitário. Por exemplo, a Linthorpe Surgery funciona de segunda a sexta-feira das 8:30 às 18:00, com uma hora de almoço em que a clínica fecha. ${ }^{14}$ Algumas clínicas oferecem horário estendido, como é o caso do Marsh House Medical Practice, em Billingham, em que nas segundas e terças-feiras a clínica permanece aberta até as 19:30.24

O horário estendido está atrelado ao contrato, como parte do pagamento adicional (enhanced services), ou seja, um incentivo financeiro para as clínicas ampliarem o acesso aos serviços. Quando a clínica está fechada, os ingleses ofertam o out of hour services. São dois os números telefônicos: o 111 para situações que não são tão graves e o 999 para atendimentos de emergência.

O 111 funciona para aconselhamento ou esclarecimento de alguma queixa para a qual a pessoa necessite orientação profissional. Funciona também como um serviço de triagem, que avalia a gravidade do caso e fornece uma orientação adequada, podendo solicitar que procure o GP no dia seguinte ou mesmo deslocar um médico até o endereço do paciente e, dependendo da gravidade, deslocar o serviço de ambulância.

\section{Estrutura da sessão clínica}

As consultas na APS inglesa estão formatadas para 10 minutos, com alguns "slots" bloqueados para eventuais atrasos (catch up time). Cada sessão clínica (clinical session) tem uma duração de quatro horas e 10 minutos. Como as consultas presenciais estão formatadas em 10 minutos e as consultas por telefone em cinco minutos, nas três primeiras horas um GP consegue atender, em média, 16 pessoas. A hora remanescente está, geralmente, dividida em visitas domiciliares e demandas burocráticas resultantes da atividade clínica, tais como: analisar resultados de exames, solicitar alguns exames, encaminhamentos, redigir laudos e atestados relativos à seguridade social, solicitar visita das enfermeiras distritais, etc. 
Em algumas clínicas é possível agendar consultas de 20 minutos, mas o paciente necessita solicitar com antecedência. As recepcionistas costumam ter uma lista de pacientes em crise "crises list of patients", que é atualizada mensalmente. Esses pacientes têm preferência no acesso aos médicos que estão fazendo seu acompanhamento ou conhecem sua situação clínica.

\section{Recepção}

O centro operacional na interface com os usuários dos serviços de APS inglesa é a recepção. Muitas das recepcionistas estão nessa função há vários anos, criando uma longitudinalidade na relação com os pacientes. São profissionais com treinamento específico para desempenhar essa função. A maioria das demandas são resolvidas por meio dessas profissionais. Quase a totalidade do agendamento é feita por telefone. Na verdade, grande parte do trabalho das recepcionistas ocorre via telefone. Este setor funciona mais como um call centre do que como uma recepção tradicional. Algumas das atividades desempenhadas pelas recepcionistas estão descritas abaixo:

(a) Renovação de prescrição: quando os pacientes solicitam renovação de receitas, as recepcionistas entram no prontuário do paciente e imprimem as prescrições previamente autorizadas pelos clínicos. As prescrições ficam disponíveis para que qualquer um dos médicos da clínica as assine durante o trabalho. Em 48 horas ficam disponíveis para que os pacientes as retirem.

(b) Resultado de exames: por telefone as recepcionistas informam ativamente aos pacientes sobre resultados de exame. Por exemplo, a recepcionista liga dizendo que o médico já avaliou os exames que resultaram normais e que não precisa vir consultar; ou ao contrário, que os exames já estão prontos, que o médico gostaria de conversar sobre os resultados e então, reserva um horário na agenda do GP.

(c) Busca ativa: os funcionários administrativos elaboram uma lista de pacientes que necessitam comparecer à clínica durante o mês vigente para revisão clínica. Essa lista é enviada às recepcionistas que, por telefone, fazem o convite aos pacientes e oferecem o agendamento que melhor lhes convém.

(d) Escuta qualificada: quando os pacientes telefonam solicitando uma consulta médica, a recepção faz uma pequena triagem e, dependendo do motivo da consulta, oferece a possibilidade de consultar com a enfermagem. As recepcionistas costumam ter uma lista das atividades e/ou procedimentos que a enfermeira é capaz de desempenhar. Elas também podem oferecer uma consulta por telefone, caso o paciente tenha dúvidas e requeira alguma orientação.

(e) Visita domiciliar: toda a demanda por visita domiciliar passa pela recepção e, geralmente, é redirecionada para uma enfermeira avaliar a real necessidade, pois dependendo da demanda essa visita pode ser realizada por um GP, Nurse Practitioner (NP), Practice Nurse (PN) ou uma enfermeira distrital.

(f) Demandas dos profissionais da clínica: as recepcionistas são o elo entre as equipes de saúde e os usuários. Assim, se um GP necessita rever um paciente ele aciona a recepção, que entra 
em contato com o usuário convidando-o para comparecer à clínica. São inúmeras as atividades nas quais a recepção está diretamente envolvida, desde o simples contato com o paciente até o apoio à vigilância em saúde via busca ativa de pacientes que necessitam comparecer à consulta clínica.

\section{Equipe de enfermagem}

AAPS britânica é bem abrangente e resolutiva. Dentro do espectro assistencial e de cuidado, o papel da enfermagem se destaca pelo grau de resolutividade. Isso faz com que a pressão assistencial sobre os médicos seja diluída, tanto na demanda espontânea quanto nas linhas de cuidado. As enfermeiras $P N$ podem se qualificar em linhas de cuidados específicos, tais como manejo de pacientes com asma/DPOC, problemas cardiovasculares (hipertensão, controle do TAP), diabetes, saúde da mulher (contracepção, preventivo do colo de útero), e assim por diante.

Geralmente, as clínicas da APS costumam contratar de uma a duas enfermeiras NPs. As NPs são um intermediário entre o GP e as PNs. Elas têm alto grau de autonomia. Podem atender a uma série de "minor illnesses" com grande autonomia prescritiva. A lista de condições que podem ser avaliadas e tratadas pelas NPs é vasta, abrangendo desde cistites, dores de cabeça, dores abdominais e musculoesqueléticas até a realização de pequenos procedimentos, tais como cauterização de lesões cutâneas, remoção de cerume do ouvido e pequenos procedimento cirúrgicos. Isso libera, em certa medida, os GPs para lidarem com pacientes com quadros indiferenciados, mais complexos e o crescente fenômeno da multimorbidade. ${ }^{25}$

\section{Sistema de informação e vigilância em saúde}

O contrato de 2004 estimulou financeiramente a adoção de prontuários eletrônicos na APS em todo o Reino Unido. Sem um sistema de informação robusto não seria possível a realização das atividades de vigilância em saúde exigidas pelo modelo de pagamento por desempenho. Todas as linhas de cuidado passaram a ser realizadas dentro de protocolos que geram os códigos exigidos para cada atividade de cuidado em saúde.

Nesse sentido, a organização do processo de trabalho visa garantir atividades programáticas (linhas de cuidado) em equilíbrio com a demanda por atendimento clínico no mesmo dia. Dessa forma, as reuniões de vigilância são organizadas com base nos dados produzidos pelo sistema de informação. É possível saber quantas e quais mulheres em idade-alvo para o rastreio do câncer do colo uterino necessitam ser convidadas a comparecer à clínica; quantas e quais pessoas não foram vacinadas contra a gripe; quantas e quais pessoas estão com a pressão arterial fora dos parâmetros aceitáveis e assim por diante.

Comumente, uma equipe composta pelo gerente geral e seus auxiliares administrativos, uma NP e um GP são responsáveis por estar à frente do levantamento e monitoramento dos dados junto à equipe de funcionários administrativos. Periodicamente, eles se reúnem para uma discussão pontual e tomada de decisões sobre as metas a serem alcançadas naquele momento. Os dados compilados e as respectivas ações propostas são apresentados à equipe para que se consiga alcançar as metas de cuidado assistencial e/ou cobertura vacinal. Assim, os profissionais da clínica refletem juntos fazendo os ajustes necessários às estratégias trazidas pela equipe e os desafios a serem superados para melhorar a vigilância em saúde. 
Em relação à demanda espontânea, há uma tendência de se criar uma via de comunicação on-line com os pacientes, de modo a facilitar o acesso a alguns serviços. Todas as clínicas têm uma webpage com as principais informações que incluem: (a) feed de notícias; (b) agendamentos; (c) horário de funcionamento; (d) serviços disponíveis; (e) renovação de receitas; (f) como se registrar na clínica; (g) equipe de saúde; e (h) como entrar em contato.

Cada vez mais os usuários do sistema estão migrando para uma relação on-line com as clínicas. Por exemplo, pode-se fazer via on-line o agendamento, ${ }^{26}$ solicitar a renovação de medicação de uso contínuo ${ }^{27}$ e compartilhar o prontuário com quem o paciente desejar. ${ }^{28} \mathrm{Na}$ webpage "Patient Access" o leitor deste artigo pode utilizar as respectivas referências dos tutoriais e navegar para ter uma ideia de como esses processos ocorrem. Também pode acessar as webpages dos grupos médicos utilizados como exemplo para que tenha uma melhor compreensão do que foi aqui brevemente descrito.

\section{Considerações finais}

A construção do welfare state no pós-guerra resultou em um grau maior de cidadania e de direitos humanos para a população britânica. O estado passou a ser o principal provedor dos serviços de saúde, originalmente com pouca influência do setor suplementar. As mudanças contratuais tornaram o NHS mais susceptível às influências do mercado, das corporações farmacêuticas e de biotecnologia em saúde.

É difícil transportar alguns dos avanços dessa sociedade para o Brasil. Por exemplo, o trabalhador britânico pode se autoatestar, i.e., emitir seu próprio atestado de afastamento do trabalho por motivo de saúde, desde que não ultrapasse sete dias (Seven days off sick or less). ${ }^{14}$ Não existe limite anual para o autoatestado. Espera-se uma relação madura entre empregado e empregador. Esse contexto reflete um modelo de seguridade social que influencia a dinâmica do cotidiano da APS.

A APS inglesa se caracteriza por um ambiente complexo de cuidados clínicos à saúde. Ela se realiza em um contexto de apoio assistencial proveniente tanto do distrito sanitário (enfermeiras distritais, enfermeiras obstetras e enfermeiras que fazem a puericultura) quanto do terceiro setor, a exemplo dos cuidados paliativos.

Todavia, pode-se ressaltar alguns pontos que favorecem o acesso nas clínicas da APS inglesa, tais como: (a) proporção população/MFC abaixo de 2000 pessoas; (b) grande número de pessoal administrativo e recepcionistas no manejo da informação e na interface com os usuários; (c) maior autonomia assistencial da equipe de enfermagem, e (d) sistema de informatização robusto, voltado tanto para as necessidades assistenciais e de vigilância em saúde quanto para a interface com os usuários.

A profissionalização do acesso na APS exige um contingente maior de recursos humanos, sem os quais a qualidade assistencial ficará certamente prejudicada. No Brasil, a ESF necessita percorrer caminho semelhante para um equilíbrio entre cuidados à demanda espontânea e ações programáticas em saúde.

\section{Contribuição dos autores}

Concepção e delineamento do estudo: AHN. Aquisição, análise e interpretação dos dados: AHN. Redação preliminar: AHN. Revisão crítica da versão preliminar: AHN. O autor aprovou a versão final e concordou com prestar contas sobre todos os aspectos do trabalho. 


\title{
Conflito de interesses
}

\author{
Declara não haver.
}

\section{Referências}

1. Tesser CD, Norman AH. Repensando o acesso ao cuidado na Estratégia Saúde da Família. Saúde Soc. 2014;23(3):869-83. https://doi.org/10.1590/S0104-12902014000300011

2. Norman AH, Tesser CD. Acesso ao cuidado na Estratégia Saúde da Família: equilíbrio entre demanda espontânea e prevenção/promoção da saúde. Saúde Soc. 2015;24(1):165-79. https://doi.org/10.1590/S0104-12902015000100013

3. Norman AH, Russell AJ, Merli C. The Quality and Outcomes Framework: Body commodification in UK general practice. Soc Sci Med. 2016;170:77-86. https://doi.org/10.1016/j.socscimed.2016.10.009

4. McCarthy M. Health system report ranks UK first, US last. BMJ. 2014;348:g4080. https://doi.org/10.1136/bmj.g4080

5. The NHS: A Difficult Beginning. [YouTube]. [acesso 2018 Dez 16]. Disponível em: https://www.youtube.com/watch?v=-ywP8wjfOx4\&t=177s

6. Gray DJ. General practitioners and the independent contractor status. J R Coll Gen Pract. 1977;27(185):746-52.

7. Starfield B, Shi L, Macinko J. Contribution of primary care to health systems and health. Milbank Q. 2005;83(3):457-502. https://doi.org/10.1111/j.1468-0009.2005.00409.x

8. Light DW. Universal health care: lessons from the British experience. Am J Public Health. 2003;93(1):25-30. https://doi.org/10.2105/ AJPH.93.1.25

9. The Birth of the National Health Service:Your Very Good Health - 1948 - CharlieDeanArchives [YouTube]. [acesso 2018 Dez 25]. Disponível em: https://www.youtube.com/watch?v=VFhEB3gG8HA

10. Choose Your Doctor - Trailer (1948). [YouTube]. [acesso 2018 Dez 16]. Disponível em: https://www.youtube.com/watch?v=y_FXAsDVStY

11. Maynard A, Dixon M. Should the NHS abolish the purchaser-provider split? BMJ. 2016;354:i3825. https://doi.org/10.1136/bmj.i3825

12. Filippon J, Giovanella L, Konder M, Pollock AM. "Liberalizing" the English National Health Service: background and risks to healthcare entitlement. Cad Saúde Pública. 2016;32(8):e00034716. https://doi.org/10.1590/0102-311X00034716

13. Jones R. Dismantling general practice. Br J Gen Pract. 2007;57(544):860-1. https://doi.org/10.3399/096016407782317874

14. Linthorpe Surgery. [acesso 2018 Dez 23]. Disponível em: https://www.linthorpesurgery.com/earnings.aspx

15. Morais HMM, Albuquerque MSV, Oliveira RS, Cazuzu AKI, Silva NAF. Organizações Sociais da Saúde: uma expressão fenomênica da privatização da saúde no Brasil. Cad Saúde Pública. 2018;34(1):e00194916. https://doi.org/10.1590/0102-311x00194916

16. Gorsky M. The British National Health Service 1948-2008: A Review of the Historiography. Soc Hist Med. 2008;21(3):437-60. https://doi.org/10.1093/shm/hkn064

17. An alternative guide to the new NHS in England. [YouTube]. [acesso 2018 Dez 16]. Disponível em: https://www.youtube.com/ watch?v=te4kNFmPkhQ

18. Norman $\mathrm{AH}$, Tesser CD. Obstetrizes e enfermeiras obstetras no Sistema Único de Saúde e na Atenção Primária à Saúde: por uma incorporação sistêmica e progressiva. Rev Bras Med Fam Comunidade. 2015;10(34):1-7. https://doi.org/10.5712/rbmfc10(34)1106

19. Macmillan Cancer Support. [acesso 2018 Dez 24]. Disponível em: https://www.macmillan.org.uk/

20. Rethink Mental IIIness: the mental health charity. [acesso 2018 Dez 24]. Disponível em: https://www.rethink.org/about-us

21.DESMOND: What is the DESMOND Programme? [acesso 2018 Dez 24]. Disponível em: https://www.desmond-project.org.uk/about/

22. Meade JG, Brown JS. Improving access for patients - a practice manager questionnaire. BMC Fam Pract. 2006;7:37. https://doi.org/10.1186/1471-2296-7-37

23. Norman AH, Russell AJ, Macnaughton J. The payment for performance model and its infuence on british general practitioners' principles and practice. Cad Saúde Pública. 2014;30(1):55-67. https://doi.org/10.1590/0102-311X00149912 
24. Marsh House: Medical Practice Billingham. [acesso 2018 Dez 22]. Disponível em: http://www.marshhousemedicalpractice.co.uk/index. php

25. Salisbury C. Multimorbidity: redesigning health care for people who use it. Lancet. 2012;380(9836):7-9. https://doi.org/10.1016/S01406736(12)60482-6

26. Patient Access Support Portal: Book appointment. [acesso 2018 Dez 22]. Disponível em: https://support.patientaccess.com/videos/ book-appointment

27.Patient Access Support Portal: Request repeat medication. [acesso 2018 Dez 22]. Disponível em: https://support.patientaccess.com/ videos/request-repeat-medication

28. Patient Access Support Portal: Share your medical record. [acesso 2018 Dez 22]. Disponível em: https://support.patientaccess.com/ videos/share-your-medical-record 